KEK-TH-417

NOVEMBER 1994

\title{
Recursion Relations in Liouville Gravity coupled to Ising Model satisfying Fusion Rules
}

\author{
KEN-JI HAMADA目 \\ National Laboratory for High Energy Physics (KEK), \\ Tsukuba, Ibaraki 305, Japan
}

\begin{abstract}
The recursion relations of $2 \mathrm{D}$ quantum gravity coupled to the Ising model discussed by the author previously are reexamined. We study the case in which the matter sector satisfies the fusion rules and only the primary operators inside the Kac table contribute. The theory involves unregularized divergences in some of correlators. We obtain the recursion relations which form a closed set among well-defined correlators on sphere, but they do not have a beautiful structure that the bosonized theory has and also give an inconsistent result when they include an ill-defined correlator with the divergence. We solve them and compute the several normalization independent ratios of the well-defined correlators, which agree with the matrix model results.
\end{abstract}

\footnotetext{
${ }^{1}$ E-mail address : hamada@theory.kek.jp
} 


\section{Introduction}

In conformal gauge, 2D quantum gravity is described as the Liouville theory coupled to matter field [1-11]. The Liouville field gives the gravitational dressing to ensure the general covariance. As a matter sector we take a conformal field theory (CFT). In general CFT has the finite number of primary fields and they satisfy the fusion rules [12]. However, in the matrix model approach the violation of the fusion rules is observed [13, 14. This problem is overcome by using the bosonized fields for the matter sector [6, 7]. In this case, as discussed by Kitazawa in ref. [6], the zero of the correlation function for the matter sector, which represents the fusion rule, is canceled with the divergence of the Liouville sector so that the combined correlaton function becomes finite. And also the primary operators outside the Kac table no longer decouple, which are identified with the gravitational descendants. We can also derive the non-linear structures called $W$ algebra constraints [15 as the Ward identities [7] of the $W_{\infty}$ symmetry [8]. The formalism can be easily extended to 2D quantum supergravity [1]. Anyway the bosonized theory has the same beautiful structures as what the matrix model has.

At the first stage of $2 \mathrm{D}$ quantum gravity [1-5], however, we mainly considered the usual CFT satisfying the fusion rules and reached the good agreement with the matrix model results. Here we reconsider the situation that the fusion rules are maintained and observe how well such a agreement is.

As a concrete example to study such an issue we take up the Liouville gravity coupled to the Ising model. The Ward identities of this system have been discussed by the author previously [5]. Here we reexamine and make clear the previous arguments and complete the calculations. Some of correlators in this theory become ill-defined due to appearance of unregularized divergences. The Ward identity is then well-defined if all correlators involved in it are well-defined, but gives an inconsistent result when an ill-defined correlator appears in the expression.

The Ward identities of the system satisfying the fusion rules are rather tricky. In the bosonized theory there exists the BRST invariant $W_{\infty}$ current [8, 10, but in the non-bosonized theory such a current dose not exist. So we here use a non-primary current like $\partial \phi$, where $\phi$ is the Liouville field, which leads to a complicated recursion relation. The derived recursion relation has a similar structure to the $\mathrm{W}$ algebra constraint [15]. We can regard it as a deformed $\mathrm{W}$ algebra constraint due to keeping the fusion rules and 
setting the gravitational descendants located outside Kac table decoupled.

A closed set of recursion relations on sphere is obtained. They have the three independent parameters related to the normalizations of three scaling operators and explicitely depend on how to assign curvature singularities which are here put on the scaling operators. But the solutions of them are independent of how to choose the assignment. We obtain some solutions and calculate the normalization independent ratios. The results agree with those calculated in the other methods [13, 14, 6].

\section{Quantum Liouville Theory coupled to Ising Model}

Two dimensonal quantum gravity is defined through the functional integrations over metric tensor of $2 \mathrm{D}$ surface $g_{\alpha \beta}$ and matter fields. After fixing the reparametrization invariance in conformal gauge $g=\mathrm{e}^{\alpha_{I} \phi} \hat{g}$, where $\phi$ is the Liouville field and $\hat{g}$ is the background metric, the theory is expressed as the combined Liouville, matter and ghosts system defined by the action $S=S^{L}+S^{M}+S^{G}$. Here $S^{L}$ is the Liouville one

$$
S^{L}=\frac{1}{8 \pi} \int d^{2} z \sqrt{\hat{g}}\left(\hat{g}^{\alpha \beta} \partial_{\alpha} \phi \partial_{\beta} \phi+Q \hat{R} \phi\right)+\mu \int d^{2} z \mathrm{e}^{\alpha_{I} \phi}
$$

and $S^{M}$ and $S^{G}$ are the matter and the $b c$-ghosts ones. In this paper we argue only the Ising model as a matter sector for simplicity. In this case we can derive the concrete results. The parameters $Q$ and $\alpha_{I}$ are then

$$
Q=\frac{7}{\sqrt{6}}, \quad \alpha_{I}=\frac{3}{\sqrt{6}} .
$$

The Ising model has the three primary states with conformal weights $\Delta=0, \frac{1}{16}, \frac{1}{2}$. The fusion rules, or operator product expansions (OPE) of the primary fields are

$$
\begin{aligned}
& \varepsilon(z, \bar{z}) \varepsilon(0,0)=\frac{1}{|z|^{2}} \\
& \varepsilon(z, \bar{z}) \sigma(0,0)=\frac{C_{\varepsilon \sigma \sigma}}{|z|} \sigma(0,0), \\
& \sigma(z, \bar{z}) \sigma(0,0)=\frac{1}{|z|^{1 / 4}}+C_{\varepsilon \sigma \sigma}|z|^{3 / 4} \varepsilon(0,0),
\end{aligned}
$$


where $\varepsilon(z, \bar{z})$ is the energy density operator with weight $(\Delta, \bar{\Delta})=\left(\frac{1}{2}, \frac{1}{2}\right)$ and $\sigma(z, \bar{z})$ is the spin field with weight $\left(\frac{1}{16}, \frac{1}{16}\right)$. The OPE coefficient is given by $C_{\varepsilon \sigma \sigma}=\frac{1}{2}[16]$. Throughout paper we keep the above OPE's.

The physical scaling operators are now given by dressing the matter primary fields $I, \sigma$ and $\varepsilon$, where $I$ denotes the identity, which are

$$
\begin{aligned}
& O_{I}=\int d^{2} z \mathrm{e}^{\alpha_{I} \phi(z, \bar{z})}, \quad O_{\sigma}=\int d^{2} z \mathrm{e}^{\alpha_{\sigma} \phi(z, \bar{z})} \sigma(z, \bar{z}), \\
& O_{\varepsilon}=\int d^{2} z \mathrm{e}^{\alpha_{\varepsilon} \phi(z, \bar{z})} \varepsilon(z, \bar{z}),
\end{aligned}
$$

where $\alpha_{\sigma}=\frac{5}{2 \sqrt{6}}$ and $\alpha_{\varepsilon}=\frac{1}{\sqrt{6}}$. $\alpha_{I}$ is given in eq.(2.2). For the sake of subsequent discussion we introduce the notations

$$
O_{a}(z, \bar{z})=\bar{c}(\bar{z}) c(z) V_{a}(z, \bar{z}), \quad O_{a}=\int d^{2} z V_{a}(z, \bar{z}),
$$

where $a=I, \sigma, \varepsilon$.

Let us define the correlation functions of the Liouville gravity. According to the argument of Goulian-Li in ref. [G], we first integrate over the zero mode of the Liouville field. Then the correlation function is expressed by the free field one

$$
\ll \mathcal{O} \gg_{g}=\kappa^{-\chi} \mu^{s} \frac{\Gamma(-s)}{\alpha_{I}}<\mathcal{O}\left(O_{I}\right)^{s}>_{g},
$$

where $g$ is genus of $2 \mathrm{D}$ surface and $\chi=2-2 g$. The $\Gamma$-function comes from the zero mode integral of $\phi$. For

$$
\mathcal{O}=\prod^{n_{1}} O_{I} \prod^{n_{2}} O_{\sigma} \prod^{n_{3}} O_{\varepsilon}
$$

$s$ is given by

$$
\begin{aligned}
s & =\frac{1}{\alpha_{I}}\left[\frac{Q}{2} \chi-n_{1} \alpha_{I}-n_{2} \alpha_{\sigma}-n_{3} \alpha_{\varepsilon}\right] \\
& =\frac{7}{6} \chi-n_{1}-\frac{5}{6} n_{2}-\frac{1}{3} n_{3} .
\end{aligned}
$$

The expression is convergent for $s<0$, while for $s \geq 0$ the integral diveges. But the $\Gamma$-function can analytically continue to the region $s>0$ ( $s \neq$ integer $)$ so that the expression is considered as a regularized form for $s>0$. This ensures that the correlator satisfies the relation $-\frac{\partial}{\partial \mu} \ll \mathcal{O} \gg_{g}=\ll O_{I} \mathcal{O} \gg_{g}$ 
even though $s>0\left(s \neq \mathbf{Z}_{+}\right)$. For the cases that $s$ is zero or positive integer the correlation function becomes ill-defined. To overcome this problem we have to go to the bosonized theory perturbed by the cosmological constant operator and one of the screening charges for matter sector [7].

\section{Equation of Motion}

We first consider the current $\partial \phi(z)$. The Ward identity is then

$$
\int d^{2} z \bar{\partial} \ll \partial \phi(z) \mathcal{O} \gg_{g}=0
$$

The OPE between $\partial \phi$ and the scaling operator $O_{a}(a=I, \sigma, \varepsilon)$ is naively caluculated as $\partial \phi(z) O_{a}(w)=-\frac{\alpha_{a}}{z-w} O_{a}(w)$.

If the operator $\partial \phi$ were primary, there would be no problem. However it is not primary, which is subject to $L_{1} \cdot \partial \phi=Q$ and $L_{n} \cdot \partial \phi=0(n \geq 2)$, such that it is transformed as

$$
\partial \phi(z)=\left(\frac{\partial z^{\prime}}{\partial z}\right)\left[\partial^{\prime} \phi^{\prime}\left(z^{\prime}\right)-\frac{Q}{2} \frac{\partial^{2} z}{\partial z^{\prime 2}} \frac{\partial z^{\prime}}{\partial z}\right] .
$$

Then we have to pay attention to the curvature singularity. Here we consider the case that the background metric which is almost flat except for the $\delta$ function singularities at the positions of the scaling operators as [4]

$$
\frac{1}{4 \pi} \sqrt{\hat{g}} \hat{R}=\sum_{a} \nu_{a} \delta^{2}\left(z-z_{a}\right)
$$

such that $\sum_{a} \nu_{a}=\chi$, where $\chi=2-2 g$ is the Euler number. We then do not assign the curvature on $\partial \phi$ and the potential term.

When we calculate the OPE we need to smooth out the curvature singularity in the neighborhood of the position of the scaling operator, which can be carried out by using the transformation

$$
z-z_{a}=\left(z^{\prime}-z_{a}^{\prime}\right)^{1-\nu_{a}}, \quad \text { or } \quad d z d \bar{z}=\frac{d z^{\prime} d \bar{z}^{\prime}}{\left|z^{\prime}-z_{a}^{\prime}\right|^{2 \nu_{a}}} .
$$

After going to the smooth $z^{\prime}$-frame, the OPE between $\partial \phi(z)$ and $O_{a}\left(z_{a}, \bar{z}_{a}\right)$ is calculated as follows:

$$
d z \partial \phi(z) O_{a}\left(z_{a}, \bar{z}_{a}\right)=d z^{\prime}\left(\partial^{\prime} \phi^{\prime}\left(z^{\prime}\right)+\frac{Q}{2} \nu_{a} \frac{1}{z^{\prime}-z_{a}^{\prime}}\right) O_{a}^{\prime}\left(z_{a}^{\prime}, \bar{z}_{a}^{\prime}\right)
$$




$$
=\left(-\alpha_{a}+\frac{Q}{2} \nu_{a}\right) d z^{\prime} \frac{1}{z^{\prime}-z_{a}^{\prime}} O_{a}^{\prime}\left(z_{a}^{\prime}, \bar{z}_{a}^{\prime}\right) .
$$

The derivative $\bar{\partial}$ in eq.(3.1) picks up the OPE singularity. For the case (2.7) we get the following expression:

$$
\left(\frac{Q}{2} \chi-n_{1} \alpha_{I}-n_{2} \alpha_{\sigma}-n_{3} \alpha_{\varepsilon}-s \alpha_{I}\right) \ll \mathcal{O} \gg_{g}=0
$$

Here we use the relation $\sum_{a} \nu_{a}=\chi$. This equation is trivial, which nothing but say that $s$ is given by eq.(2.8). This equation stands for that the Liouville field satisfy the equation of motion

$$
\bar{\partial} \partial \phi(z, \bar{z})=\pi \alpha_{I} \mu \mathrm{e}^{\alpha_{I} \phi(z, \bar{z})}
$$

in the interaction picture $\ll \cdots \gg$.

Here the curvature treatment does well for arbitrary genus, but in general it breaks down for higher genus because the coordinate transformation (3.4) is not globally well-defined for higher genus. In the sequence we consider only the sphere topology.

\section{Recursion Relations}

In this section we consider more complicated Ward identities which give informations for correlation functions. Let us introduce the operator of the form $W(z, \bar{z})=R(z) \bar{B}(\bar{z})$, where $R(z)$ has the conformal weight 1 and $B(z)$ has the weight 0 and they have the same Liouville charge. As non-trivial operators of $B(z)$, we here consider the following BRST invariant ones:

$$
B_{\sigma}(z)=\left(b c \sigma-\frac{4}{2 \sqrt{6}} \partial \phi \sigma-\frac{4}{3} \partial \sigma\right) \mathrm{e}^{-\frac{3}{2 \sqrt{6}} \phi(z)}
$$

and

$$
B_{\varepsilon}(z)=\left(b c \varepsilon-\frac{3}{2 \sqrt{6}} \partial \phi \varepsilon-\frac{3}{4} \partial \varepsilon\right) \mathrm{e}^{-\frac{4}{2 \sqrt{6}} \phi(z)},
$$

which correspond to the ring elements in the bosonized theory [8-10]. The divergence of $W(z, \bar{z})$ is

$$
\bar{\partial} W(z, \bar{z})=R(z)\left\{\bar{Q}_{B R S T},\left[\bar{b}_{-1}, \bar{B}(\bar{z})\right]\right\},
$$


where we use the fact that $\bar{B}(\bar{z})$ is the BRST invariant operator. The antiholomorphic part is BRST-trivial so that the operator $W(z, \bar{z})$ plays a role of current.

The operator $R(z)$ has the same Liouville charge as $B(z)$ and the conformal weight 1 . Therefore the partners of $B_{\sigma}(z)$ and $B_{\varepsilon}(z)$ should include the two derivatives. Fitting in our previous work [5], we here take up the following forms:2

$$
R_{\sigma}(z)=\left(\frac{7}{3} \partial^{2} \phi-\frac{4}{\sqrt{6}}(\partial \phi)^{2}\right) \mathrm{e}^{-\frac{3}{2 \sqrt{6}} \phi} \sigma(z)
$$

and

$$
R_{\varepsilon}(z)=\left(\frac{7}{4} \partial^{2} \phi-\frac{3}{\sqrt{6}}(\partial \phi)^{2}\right) \mathrm{e}^{-\frac{4}{2 \sqrt{6}} \phi} \varepsilon(z) .
$$

These are not the primary operators. In the bosonized theory there exist the primary states, callled the discrete states, which form the $W_{\infty}$ algebra. In this paper, however, we do not use the bosonization for the matter sector. Then such a primary state does not exist so that in this theory the Ward identities do not have the beautiful structures like the $\mathrm{W}$ algebra constraints. As discussed below the Ward identities have more complicated forms and we cannot argue them beyond the topology of sphere.

The complexity comes from the non-tensor transformation property of $R(z)$. The operator $R_{\sigma}(z)$ satisfies the equations

$$
\begin{aligned}
& L_{1} \cdot R_{\sigma}(z)=S_{\sigma}(z), \quad L_{2} \cdot R_{\sigma}(z)=\frac{9}{4} T_{\sigma}(z), \\
& L_{1} \cdot S_{\sigma}(z)=-\frac{17}{8} T_{\sigma}(z),
\end{aligned}
$$

$L_{n} \cdot R_{\sigma}(z)=0(n \geq 3)$ and $L_{n} \cdot S_{\sigma}(z)=0(n \geq 2)$, where $L_{n}=L_{n}^{L}+L_{n}^{M}+L_{n}^{G}$ and

$$
L_{n}^{L, M, G} \cdot A(z)=\oint_{C_{z}} \frac{d y}{2 \pi i}(y-z)^{n+1} T^{L, M, G}(y) A(z)
$$

for some operator $A(z)$. Here $T^{L, M, G}(z)$ are the energy-momentum tensors for the Liouville, the matter and the ghost sectors respectively. These imply

\footnotetext{
${ }^{2}$ We may use the other type of the operator because we here do not impose the primary conditions.
} 
that $R_{\sigma}(z)$ is transformed as

$$
\begin{aligned}
R_{\sigma}(z)=\left(\frac{\partial z^{\prime}}{\partial z}\right)\left[R_{\sigma}^{\prime}\left(z^{\prime}\right)\right. & -\frac{1}{2} \frac{\partial^{2} z}{\partial z^{\prime 2}} \frac{\partial z^{\prime}}{\partial z} S_{\sigma}^{\prime}\left(z^{\prime}\right)-\frac{3}{8} \frac{\partial^{3} z}{\partial z^{\prime 3}} \frac{\partial z^{\prime}}{\partial z} T_{\sigma}^{\prime}\left(z^{\prime}\right) \\
& \left.+\frac{19}{64}\left(\frac{\partial^{2} z}{\partial z^{\prime 2}}\right)^{2}\left(\frac{\partial z^{\prime}}{\partial z}\right)^{2} T_{\sigma}^{\prime}\left(z^{\prime}\right)\right]
\end{aligned}
$$

where $S_{\sigma}(z)$ and $T_{\sigma}(z)$ are the operators with weights 0 and -1 respectively defined by

$$
S_{\sigma}(z)=L_{-1}^{L} \cdot T_{\sigma}(z), \quad T_{\sigma}(z)=\frac{80}{3 \sqrt{6}} \mathrm{e}^{-\frac{3}{2 \sqrt{6}} \phi} \sigma(z)
$$

which is transformed as

$$
\begin{aligned}
& S_{\sigma}(z)=S_{\sigma}^{\prime}\left(z^{\prime}\right)+\frac{17}{16} \frac{\partial^{2} z}{\partial z^{\prime 2}} \frac{\partial z^{\prime}}{\partial z} T_{\sigma}^{\prime}\left(z^{\prime}\right), \\
& T_{\sigma}(z)=\left(\frac{\partial z^{\prime}}{\partial z}\right)^{-1} T_{\sigma}^{\prime}\left(z^{\prime}\right) .
\end{aligned}
$$

The transformation law of the operator $R_{\varepsilon}(z)$ is also given as follows:

$$
\begin{aligned}
R_{\varepsilon}(z)=\left(\frac{\partial z^{\prime}}{\partial z}\right)\left[R_{\varepsilon}^{\prime}\left(z^{\prime}\right)\right. & -\frac{1}{2} \frac{\partial^{2} z}{\partial z^{\prime 2}} \frac{\partial z^{\prime}}{\partial z} S_{\varepsilon}^{\prime}\left(z^{\prime}\right)-\frac{17}{36} \frac{\partial^{3} z}{\partial z^{\prime 3}} \frac{\partial z^{\prime}}{\partial z} T_{\varepsilon}^{\prime}\left(z^{\prime}\right) \\
& \left.+\frac{1}{3}\left(\frac{\partial^{2} z}{\partial z^{\prime 2}}\right)^{2}\left(\frac{\partial z^{\prime}}{\partial z}\right)^{2} T_{\varepsilon}^{\prime}\left(z^{\prime}\right)\right]
\end{aligned}
$$

where

$$
\begin{aligned}
& L_{1} \cdot R_{\varepsilon}(z)=S_{\varepsilon}(z), \quad L_{2} \cdot R_{\varepsilon}(z)=\frac{17}{6} T_{\varepsilon}(z) \\
& L_{1} \cdot S_{\varepsilon}(z)=-3 T_{\varepsilon}(z)
\end{aligned}
$$

and

$$
S_{\varepsilon}(z)=L_{-1}^{L} \cdot T_{\varepsilon}(z), \quad T_{\varepsilon}(z)=\frac{33}{2 \sqrt{6}} \mathrm{e}^{-\frac{4}{2 \sqrt{6}} \phi} \varepsilon(z)
$$

\subsection{Ward identities for $W_{\sigma}$}

We first consider the Ward identities for the current $W_{\sigma}(z, \bar{z})$, which is

$$
\int d^{2} z \bar{\partial} \ll W_{\sigma}(z, \bar{z}) \mathcal{O} \gg_{0}=0
$$


Let us calculate the OPE between the current and the scaling operators. As discussed in Sect.3 the curvature singularities are assigned on the scaling operators and we treat them with care. By transforming into the non-singular frame we get the following OPE:

$$
\begin{aligned}
& d z W_{\sigma}(z, \bar{z}) O_{\sigma}(w, \bar{w}) \\
& =d z^{\prime}\left[R_{\sigma}^{\prime}\left(z^{\prime}\right)+\frac{\nu}{2} \frac{1}{z^{\prime}-w^{\prime}} S_{\sigma}^{\prime}\left(z^{\prime}\right)-\frac{5 \nu^{2}+24 \nu}{64\left(z^{\prime}-w^{\prime}\right)^{2}} T_{\sigma}^{\prime}\left(z^{\prime}\right)\right] \\
& \quad \times \bar{B}_{\sigma}^{\prime}\left(\bar{z}^{\prime}\right) O_{\sigma}^{\prime}\left(w^{\prime}, \bar{w}^{\prime}\right) \\
& =d z^{\prime} \frac{1}{z^{\prime}-w^{\prime}}\left(-\frac{10}{9 \sqrt{6}}\right) C_{\varepsilon \sigma \sigma}\left(1-\nu-\frac{5}{4} \nu^{2}\right) O_{\varepsilon}^{\prime}\left(w^{\prime}, \bar{w}^{\prime}\right),
\end{aligned}
$$

where $\nu$ is the curvature singularity assigned on $O_{\sigma}$. In order to smooth away it we use the coordinate transformation (3.4) and the transformation law of $R_{\sigma}(z)$ (4.9). The OPE's of the current with $O_{I}$ and $O_{\varepsilon}$ do not give any contribution.

The derivative $\bar{\partial}$ in eq.(4.16) picks up the OPE singularity calculated above and also produce the following correlator:

$$
\ll \int d^{2} z \bar{\partial} W_{\sigma}(z, \bar{z}) \mathcal{O} \gg_{0},
$$

where the anti-holomorphic part of $\bar{\partial} W_{\sigma}(z, \bar{z})$ is BRST-trivial (4.3). So this correlator usually would vanish. However, as discussed in the previous papers [4, 青, 7], the boundary of moduli space is now dangerous and this correlator gives an anomalous contribution.

Using the factorization formula, we can calculate such a contribution. We need to evaluate the following expression:

$$
\begin{aligned}
\kappa^{2} \sum_{N=1}^{\infty} \sum_{\Delta} \int_{-\infty}^{\infty} & \frac{d p}{2 \pi} \ll \mathcal{O}_{1} \int_{|z| \leq 1} d^{2} z \bar{\partial} W_{\sigma}(z, \bar{z}) \\
& \times D\left|-p, \Delta ; N \gg_{0} \ll p, \Delta ; N\right| \mathcal{O}_{2} \gg_{0}
\end{aligned}
$$

where $\Delta=0, \frac{1}{16}$ and $\frac{1}{2}$. $D$ is the propergator. The intermediate state is the normalizable eigenstate of the Hamiltonian $H=L_{0}+\bar{L}_{0}$ with the eigenvalue $p^{2}+\frac{Q^{2}}{4}+2(\Delta+N-1)$. For $N=0$ it is given by

$$
\left|p, \Delta>=\mathrm{e}^{\left(i p+\frac{Q}{2}\right) \phi(0,0)} \Phi_{\Delta}(0,0)\right| 0>_{L, M} \otimes \bar{c}_{1} c_{1} \mid 0>_{G},
$$


where $\Phi_{\Delta}=I, \sigma, \varepsilon . N \neq 0$ states are their descendants. The normalization is

$$
\ll p^{\prime}, \Delta^{\prime} ; N^{\prime} \mid p, \Delta ; N \gg_{0}=\kappa^{-2} 2 \pi \delta\left(p+p^{\prime}\right) \delta_{\Delta, \Delta^{\prime}} \delta_{N, N^{\prime}}
$$

The zero mode integral of the Liouville field now produces the $\delta$-function.

The propagator is defined by

$$
D=\int_{|z| \leq 1} \frac{d^{2} z}{|z|^{2}} z^{L_{0}} \bar{z}^{\bar{L}_{0}}=2 \pi\left(\frac{1}{H}-\lim _{\tau \rightarrow \infty} \frac{1}{H} \mathrm{e}^{-\tau H}\right),
$$

where we introduce the regulator at $|z|=\mathrm{e}^{-\tau}$. The last term stands for the boundary of moduli space. Since the BRST charge commutes with the Hamiltonian there is no contribution from $1 / H$ term. The boundary term is now dangerous. So we evaluate the following quantity carefully:

$$
\begin{aligned}
\lim _{\tau \rightarrow \infty} \kappa^{2} \sum_{\Delta} \int_{-\infty}^{\infty} \frac{d p}{2 \pi} \int_{\mathrm{e}^{-\tau} \leq|z| \leq 1} d^{2} z \ll \mathcal{O}_{1} R_{\sigma}(z)\left[\bar{b}_{-1}, \bar{B}_{\sigma}(\bar{z})\right] \\
\quad \times \bar{Q}_{B R S T}\left(-\frac{2 \pi}{H} \mathrm{e}^{-\tau H}\right)\left|-p, \Delta ; N \gg_{0} \ll p, \Delta ; N\right| \mathcal{O}_{2} \gg_{0}
\end{aligned}
$$

where we omit $N \neq 0$ modes because, as discussed below, these modes vanish at $\tau \rightarrow \infty$. Noting

$$
\bar{Q}_{B R S T}\left(-\frac{2 \pi}{H} \mathrm{e}^{-\tau H}\right)\left|-p, \Delta>=-\pi \bar{\partial} \bar{c}(0) \mathrm{e}^{-\tau H}\right|-p, \Delta>
$$

and

$$
\left[\bar{b}_{-1}, \bar{B}_{\sigma}(\bar{z})\right]=-\bar{b}(\bar{z}) \mathrm{e}^{-\frac{3}{2 \sqrt{6}} \phi(\bar{z})} \sigma(\bar{z})
$$

we obtain the expression

$$
\begin{aligned}
\lim _{\tau \rightarrow \infty} \kappa^{2} \sum_{\Delta} & \int_{-\infty}^{\infty} \frac{d p}{2 \pi} \pi C_{\sigma \Delta \Delta^{\prime}} A_{\sigma}(p, \Delta) \mathrm{e}^{-\tau\left(p^{2}+\frac{Q^{2}}{4}+2 \Delta-2\right)} \\
& \times \int_{\mathrm{e}^{-\tau} \leq|z| \leq 1} d^{2} z|z|^{2\left\{-2+\frac{3}{2 \sqrt{6}}\left(-i p+\frac{Q}{2}\right)-\frac{1}{16}-\Delta+\Delta^{\prime}\right\}} \\
& \times \ll \mathcal{O}_{1}\left|-p+i \frac{3}{2 \sqrt{6}}, \Delta^{\prime} \gg_{0} \ll p, \Delta\right| \mathcal{O}_{2} \gg_{0}
\end{aligned}
$$

where $C_{\sigma \Delta \Delta^{\prime}}$ is the OPE coefficient for the matter sector defined by eq.(2.3). When the correlator is divided into two on sphere, the curvature singularity is induced at the state $\mid-p, \Delta \gg$ to ensure that the Euler number is 2 for sphere. 
We here describe the induced curvature on the state as $\nu_{p}$. Simultaneously the curvature $2-\nu_{p}$ is induced at the state $\ll p, \Delta \mid$. The coefficient $A_{\sigma}(p, \Delta)$, which comes from the Liouville sector, is given as follows:

$$
\begin{aligned}
A_{\sigma}(p, \Delta)=\frac{7}{3} & \left(-i p+\frac{Q}{2}\right)-\frac{4}{\sqrt{6}}\left(-i p+\frac{Q}{2}\right)^{2} \\
& +\frac{10}{3} \nu_{p}\left(-i p+\frac{Q}{2}\right)-\frac{80}{3 \sqrt{6}} \frac{5 \nu_{p}^{2}+24 \nu_{p}}{64} .
\end{aligned}
$$

Changing the variable to $z=\mathrm{e}^{-\tau x+i \theta}$, where $0 \leq x \leq 1$ and $0 \leq \theta \leq 2 \pi$, the above expression is rewritten as

$$
\begin{gathered}
\lim _{\tau \rightarrow \infty} \kappa^{2} \sum_{\Delta} \int_{-\infty}^{\infty} \frac{d p}{2 \pi} \int_{0}^{1} 2 \pi \tau d x \pi C_{\sigma \Delta \Delta^{\prime}} A(p, \Delta) \\
\times \ll \mathcal{O}_{1}\left|-p+i \frac{3}{2 \sqrt{6}}, \Delta^{\prime} \gg_{0} \ll p, \Delta\right| \mathcal{O}_{2} \gg_{0} \exp \left[-\tau\left\{p^{2}+\frac{1}{24}+2 \Delta\right.\right. \\
\left.\left.+2 x\left(\frac{3}{2 \sqrt{6}}\left(-i p+\frac{Q}{2}\right)-1-\frac{1}{16}-\Delta+\Delta^{\prime}\right)\right\}\right] .
\end{gathered}
$$

Since the exponential term is highly peaked in the limit $\tau \rightarrow \infty$, the saddle point estimation becomes exact. The saddle point of the $p$ integral is $p=$ $i \frac{3}{2 \sqrt{6}} x$, so that $(4.29)$ becomes

$$
\begin{aligned}
\lim _{\tau \rightarrow \infty} & \kappa^{2} \sum_{\Delta} \pi \tau \sqrt{\frac{2 \pi}{2 \tau}} \int_{0}^{1} d x C_{\sigma \Delta \Delta^{\prime}} A\left(i \frac{3}{2 \sqrt{6}} x, \Delta\right) \\
& \times \ll \mathcal{O}_{1}\left|i \frac{3}{2 \sqrt{6}}(1-x), \Delta^{\prime} \gg_{0} \ll i \frac{3}{2 \sqrt{6}} x, \Delta\right| \mathcal{O}_{2} \gg_{0} \\
& \times \exp \left[-\tau\left\{\frac{3}{8} x^{2}-\left(\frac{3}{8}+2 \Delta-2 \Delta^{\prime}\right) x+\frac{1}{24}+2 \Delta\right\}\right] .
\end{aligned}
$$

$x$ integral is also evaluated at the saddle point

$$
x=\frac{1}{6}\left(3+16 \Delta-16 \Delta^{\prime}\right) .
$$

From the fusion rules (2.3) the possible pairs of $\left(\Delta, \Delta^{\prime}\right)$ are $\left(0, \frac{1}{16}\right),\left(\frac{1}{2}, \frac{1}{16}\right)$, $\left(\frac{1}{16}, 0\right)$ and $\left(\frac{1}{16}, \frac{1}{2}\right)$. In order to get non-vanishing contributions it is necessary that the saddle point is located within the interval $0 \leq x \leq 1$. Thus only two 
cases $\left(0, \frac{1}{16}\right)$ and $\left(\frac{1}{16}, 0\right)$ survive. For $\left(0, \frac{1}{16}\right)$ the saddle point is $x=\frac{1}{3}$ and we obtain

$$
-\pi^{2} \kappa^{2} \frac{8}{9}\left(\frac{5}{4} \nu_{p}-1\right)^{2} \ll \mathcal{O}_{1} O_{\sigma} \gg_{0} \ll O_{I} \mathcal{O}_{2} \gg_{0}
$$

For $\left(\frac{1}{16}, 0\right)$ the saddle point is $x=\frac{2}{3}$ and we get

$$
-\pi^{2} \kappa^{2} 2\left(\frac{5}{6} \nu_{p}-1\right)^{2} \ll \mathcal{O}_{1} O_{I} \gg_{0} \ll O_{\sigma} \mathcal{O}_{2} \gg_{0}
$$

Replacing $\Delta$ and $\Delta^{\prime}$ with $\Delta+N$ and $\Delta^{\prime}+N$ in the expression (4.29), we can see that the contributions from oscilation modes vanish exponentially as $\mathrm{e}^{-2 N \tau}$. Combining the results (4.32) and (4.33) we obtain the following expression:

$$
\begin{aligned}
\ll \int d^{2} z \bar{\partial} W_{\sigma}(z, \bar{z}) \prod_{a \in S} O_{a} \gg_{0}= \\
-\frac{\pi^{2} \kappa^{2}}{2} \sum_{S=X \cup Y}\left[\frac{8}{9}\left(\frac{5}{4} \nu_{p}-1\right)^{2} \ll O_{\sigma} \prod_{b \in X} O_{b} \gg_{0} \ll O_{I} \prod_{c \in Y} O_{c} \gg_{0}\right. \\
\left.\quad+2\left(\frac{5}{6} \nu_{p}-1\right)^{2} \ll O_{I} \prod_{b \in X} O_{b} \gg_{0} \ll O_{\sigma} \prod_{c \in Y} O_{c} \gg_{0}\right],
\end{aligned}
$$

where the factor $\frac{1}{2}$ in r.h.s. corrects for double counting. The induced curvature $\nu_{p}$ satisfies the relation

$$
\nu_{p}+\sum_{b \in X} \nu_{b}=2
$$

Note that $\nu_{p}$ also satisfies the relation $2-\nu_{p}+\sum_{c \in Y} \nu_{c}=2$ because of $\sum_{a \in S} \nu_{a}=2$. The first and the second terms of the r.h.s. of eq.(4.34) are symmetric under the interchange $\nu_{p} \leftrightarrow 2-\nu_{p}$.

Let us first consider the case of $\mathcal{O}=\prod^{n} O_{\sigma}$. To write down the expression we have to specify the assignment of the curvature singularity. The expression depends on the assignment, but the solution is independent of how to choose it. Here we properly assign the curvatures $\nu_{a}(a=1 \cdots n)$ on $n O_{\sigma}$ operators, where $\sum_{a=1}^{n} \nu_{a}=\chi=2$. Then we get

$$
\pi\left(-\frac{10}{9 \sqrt{6}}\right) C_{\varepsilon \sigma \sigma}\left(n-2-\frac{5}{4} \sum_{a=1}^{n} \nu_{a}^{2}\right) \ll O_{\varepsilon} \prod^{n-1} O_{\sigma} \gg_{0}
$$




$$
\begin{aligned}
& -\frac{\pi}{\sqrt{6}} \lambda_{1} \sum_{k=0}^{n}\left[2\left(\begin{array}{c}
n-2 \\
k
\end{array}\right)+\frac{8}{9}\left(\begin{array}{l}
n-2 \\
k-2
\end{array}\right)-\frac{8}{3}\left(\begin{array}{l}
n-2 \\
k-1
\end{array}\right)\right. \\
& \left.+\frac{25}{18}\left(\begin{array}{l}
n-2 \\
k-1
\end{array}\right) \sum_{a=1}^{n} \nu_{a}^{2}\right] \ll \prod O_{\sigma} \gg_{0} \ll O_{I} \prod^{n-k} O_{\sigma} \gg_{0}=0,
\end{aligned}
$$

where

$$
\lambda_{1}=\sqrt{6} \pi \kappa^{2}
$$

This equation is valid for $n \geq 3$. The $n=2$ equation is trivially satisfied due to the vanishing of the corrlators with odd numbers of $O_{\sigma}$. The $n=1$ is ill-defined as discussed in the last of Sect.2. The factorization form is given as follows. The sum over $k$ stands for choosing $k$ operators which form the set $X$ out of $n O_{\sigma}$ 's in the set $S$. Then we have the following formulas:

$$
\sum_{S=X \cup Y}=\sum_{k=1}^{n} \sum_{\{X\}_{k}},
$$

and

$$
\begin{aligned}
& \sum_{\{X\}_{k}} 1=\left(\begin{array}{l}
n \\
k
\end{array}\right), \quad \sum_{\{X\}_{k}} \sum_{a \in X} \nu_{a}=\left(\begin{array}{l}
n-1 \\
k-1
\end{array}\right) \chi, \\
& \sum_{\{X\}_{k}}\left(\sum_{a \in X} \nu_{a}\right)^{2}=\left(\begin{array}{l}
n-2 \\
k-2
\end{array}\right) \chi^{2}+\left(\begin{array}{l}
n-2 \\
k-1
\end{array}\right) \sum_{a=1}^{n} \nu_{a}^{2},
\end{aligned}
$$

where $\sum_{\{X\}_{k}}$ is taken over all possibility of choosing $k$ out of $n O_{\sigma}$ 's. Using eq.(4.35) and above formulas for sphere $\chi=\sum_{a=1}^{n} \nu_{a}=2$, we obtain the expression (4.36).

Let us next consider the the case of $\mathcal{O}=O_{\sigma} \prod^{n} O_{I}$. Here we assign the curvatures only on $n O_{I}$ operators. Then we get

$$
\begin{aligned}
& \pi^{2} \kappa^{2} \sum_{k=0}^{n}\left[2\left(\begin{array}{c}
n-2 \\
k
\end{array}\right)+\frac{8}{9}\left(\begin{array}{l}
n-2 \\
k-2
\end{array}\right)-\frac{1}{3}\left(\begin{array}{l}
n-2 \\
k-1
\end{array}\right)\right. \\
& \left.+\frac{25}{18}\left(\begin{array}{l}
n-2 \\
k-1
\end{array}\right) \sum_{a=1}^{n} \nu_{a}^{2}\right] \ll O_{\sigma} O_{\sigma} \prod^{k} O_{I} \gg_{0} \ll \prod^{n-k+1} O_{I} \gg_{0}=0,
\end{aligned}
$$

where we use $\ll O_{\varepsilon} \prod^{n} O_{I} \gg_{0}=0$. This equation is valid for $n \geq 3$. This equation is, however, rather trivial. For $n<3$ the correlator $\ll W_{\sigma} \mathcal{O} \gg_{0}$ becomes ill-defined. 


\subsection{Ward identities for $W_{\varepsilon}$}

In this subsection we consider the Ward identities for the current $W_{\varepsilon}(z, \bar{z})$. The OPE between $W_{\varepsilon}(z, \bar{z})$ and the scaling operators which contributes to the Ward identities is

$$
\begin{aligned}
& d z W_{\varepsilon}(z, \bar{z}) O_{I}(w, \bar{w}) \\
& =d z^{\prime}\left[R_{\varepsilon}^{\prime}\left(z^{\prime}\right)+\frac{\nu}{2} \frac{1}{z^{\prime}-w^{\prime}} S_{\varepsilon}^{\prime}\left(z^{\prime}\right)-\frac{5 \nu^{2}+17 \nu}{36\left(z^{\prime}-w^{\prime}\right)^{2}} T_{\varepsilon}^{\prime}\left(z^{\prime}\right)\right] \\
& \quad \times \bar{B}_{\varepsilon}^{\prime}\left(\bar{z}^{\prime}\right) O_{I}^{\prime}\left(w^{\prime}, \bar{w}^{\prime}\right) \\
& =d z^{\prime} \frac{1}{z^{\prime}-w^{\prime}}\left(-\frac{3}{16 \sqrt{6}}\right)\left(1+\frac{11}{18} \nu-\frac{55}{18} \nu^{2}\right) O_{\varepsilon}^{\prime}\left(w^{\prime}, \bar{w}^{\prime}\right),
\end{aligned}
$$

where $\nu$ is the curvature singularity assigned on $O_{I}$.

We also need to calculate the following OPE that $W_{\varepsilon}(z, \bar{z})$ and two $O_{\sigma}$ 's get together and produce $O_{I}$ :

$$
d z W_{\varepsilon}(z, \bar{z}) O_{\sigma}(0,0) \int d^{2} w V_{\sigma}(w, \bar{w})=d z^{\prime} \frac{1}{z^{\prime}} C\left(\nu_{a}, \nu_{b}\right) O_{I}^{\prime}(0,0),
$$

where $\nu_{a}$ and $\nu_{b}$ are the curvature assigned on two $O_{\sigma}$ 's. The coefficient $C\left(\nu_{a}, \nu_{b}\right)$ are calculated as follows. For the holomorphic part we get

$$
\begin{aligned}
& d z R_{\varepsilon}(z) O_{\sigma}(0) d w V_{\sigma}(w) \\
& =d z^{\prime}\left[R_{\varepsilon}^{\prime}\left(z^{\prime}\right)+\frac{1}{2}\left(\frac{\nu_{a}}{z^{\prime}}+\frac{\nu_{b}}{z^{\prime}-w^{\prime}}\right) S_{\varepsilon}^{\prime}\left(z^{\prime}\right)\right. \\
& \left.\quad-\frac{1}{36}\left(\frac{5 \nu_{a}^{2}+17 \nu_{a}}{z^{\prime 2}}+\frac{5 \nu_{b}^{2}+17 \nu_{b}}{\left(z^{\prime}-w^{\prime}\right)^{2}}+\frac{10 \nu_{a} \nu_{b}}{z^{\prime}\left(z^{\prime}-w^{\prime}\right)}\right) T_{\varepsilon}^{\prime}\left(z^{\prime}\right)\right] \\
& \times O_{\sigma}^{\prime}(0) d w^{\prime} V_{\sigma}^{\prime}\left(w^{\prime}\right),
\end{aligned}
$$

where, to go to the non-singular frame, we use transformation law of $R_{\varepsilon}(z)$ (4.13) and the coordinate transformation defined by

$$
\frac{\partial z}{\partial z^{\prime}}=\left(z^{\prime}-z_{a}^{\prime}\right)^{-\nu_{a}}\left(z^{\prime}-z_{b}^{\prime}\right)^{-\nu_{b}}
$$

with $z_{a}^{\prime}=0$ and $z_{b}^{\prime}=w^{\prime}$. After contracting all operators we get

$$
\frac{1}{24 \sqrt{6}} d z^{\prime}\left[\left(30-22 \nu_{a}-55 \nu_{a}^{2}\right) \frac{1}{z^{\prime 2}}+\left(30-22 \nu_{b}-55 \nu_{b}^{2}\right) \frac{1}{\left(z^{\prime}-w^{\prime}\right)^{2}}\right.
$$




$$
\begin{aligned}
&\left.+\left(-150+165\left(\nu_{a}+\nu_{b}\right)-110 \nu_{a} \nu_{b}\right) \frac{1}{z^{\prime}\left(z^{\prime}-w^{\prime}\right)}\right] \\
& \times z^{\prime \frac{1}{3}}\left(z^{\prime}-w^{\prime}\right)^{\frac{1}{3}} w^{\prime-\frac{2}{3}} d w^{\prime} d_{\varepsilon \sigma \sigma} O_{I}^{\prime}(0),
\end{aligned}
$$

where $C_{\varepsilon \sigma \sigma}=d_{\varepsilon \sigma \sigma} \bar{d}_{\varepsilon \sigma \sigma}$. The calculation of the anti-holomorphic part is rather simple because $\bar{B}_{\varepsilon}(\bar{z})$ is the BRST invariant operator with weight 0 such that it is transformed as a scalar. The result is

$$
\begin{aligned}
& \bar{B}_{\varepsilon}(\bar{z}) O_{\sigma}(0) d \bar{w} V_{\sigma}(\bar{w}) \\
& =\frac{1}{\bar{z}^{\prime}-\bar{w}^{\prime}} \bar{z}^{\prime \frac{1}{3}}\left(\bar{z}^{\prime}-\bar{w}^{\prime}\right)^{\frac{1}{3}} \bar{w}^{\prime-\frac{2}{3}} d \bar{w}^{\prime} \bar{d}_{\varepsilon \sigma \sigma} O_{I}^{\prime}(0) .
\end{aligned}
$$

Combining the holomorphic and the anti-holomorphic parts and carrying out the $w$-integral, we get

$$
\begin{aligned}
C\left(\nu_{a}, \nu_{b}\right)=-\frac{5}{\sqrt{6}} \pi\left(\frac{\Gamma\left(\frac{1}{3}\right)}{\Gamma\left(\frac{2}{3}\right)}\right)^{3} C_{\varepsilon \sigma \sigma} & {\left[1-\frac{77}{60}\left(\nu_{a}+\nu_{b}\right)\right.} \\
& \left.+\frac{11}{48}\left(\nu_{a}^{2}+\nu_{b}^{2}\right)+\frac{11}{12} \nu_{a} \nu_{b}\right] .
\end{aligned}
$$

To derive this expression we use the following integral formula:

$$
\begin{aligned}
I_{n} & =\int d^{2} y|y|^{-\frac{4}{3}}|1-y|^{-\frac{4}{3}}(1-y)^{n} \\
& =\pi \frac{\Gamma\left(\frac{1}{3}\right)^{2} \Gamma\left(\frac{1}{3}+n\right)}{\Gamma\left(\frac{2}{3}\right)^{2} \Gamma\left(\frac{2}{3}+n\right)} .
\end{aligned}
$$

The anomalous correlator including the operator $\bar{\partial} W_{\varepsilon}(z, \bar{z})$ is evaluated as in the same way discussed in Sect.4.1. Here we do not repeat the calculation. The result is

$$
\begin{aligned}
& \ll \int d^{2} z \bar{\partial} W_{\varepsilon}(z, \bar{z}) \prod_{a \in S} O_{a} \gg_{0} \\
& =\frac{\pi^{2} \kappa^{2}}{2} \sum_{S=X \cup Y} C_{\varepsilon \sigma \sigma} \frac{1}{48}\left[1-55\left(\nu_{p}-1\right)^{2}\right] \\
& \quad \times \ll O_{\sigma} \prod_{b \in X} O_{b} \gg_{0} \ll O_{\sigma} \prod_{c \in Y} O_{c} \gg_{0},
\end{aligned}
$$

where $\nu_{p}$ is the induced curvature which satisfies eq.(4.35). 
Let us consider the Ward identity of the type: $\mathcal{O}=\prod^{n} O_{\sigma}$. Then the curvature $\nu_{a}(a=1, \cdots n)$ are assigned on $n O_{\sigma}$ 's $(n \geq 1)$. Using the results calculated above we get the following equation:

$$
\begin{aligned}
& \pi \frac{3}{16 \sqrt{6}} \mu \ll O_{\varepsilon} \prod^{n} O_{\sigma} \gg_{0} \\
& -\frac{\pi}{\sqrt{6}} \lambda_{2} C_{\varepsilon \sigma \sigma}\left[\frac{1}{2} n(n-1)-\frac{77}{30}(n-1)+\frac{11}{6}\right. \\
& \left.\quad+\frac{11}{48}(n-3) \sum_{a=1}^{n} \nu_{a}^{2}\right] \ll O_{I} \prod^{n-2} O_{\sigma} \gg_{0} \\
& -\frac{\pi}{2 \sqrt{6}} \lambda_{1} C_{\varepsilon \sigma \sigma} \sum_{k=0}^{n}\left[\frac{54}{48}\left(\begin{array}{l}
n \\
k
\end{array}\right)-\frac{55}{48}\left(\begin{array}{l}
n-2 \\
k-1
\end{array}\right)\left(4-\sum_{a=1}^{n} \nu_{a}^{2}\right)\right] \\
& \quad \times \ll \prod^{k+1} O_{\sigma} \gg_{0} \ll \prod^{n-k+1} O_{\sigma} \gg_{0}=0,
\end{aligned}
$$

where $\lambda_{1}$ is defined by (4.37) and

$$
\lambda_{2}=5 \pi\left(\frac{\Gamma\left(\frac{1}{3}\right)}{\Gamma\left(\frac{2}{3}\right)}\right)^{3} .
$$

The first term comes from the OPE between the current and the potential $O_{I}$. To derive the second expression we use the relations $\sum_{a=1}^{n} \nu_{a}=2$ and $\sum_{a, b=1(a \neq b)}^{n} \nu_{a} \nu_{b}=4-\sum_{a=1}^{n} \nu_{a}^{2}$. This equation is defined for $n \geq 1$, but it becomes trivial for odd $n$ due to the vanishing of correlators including the odd numbers of $O_{\sigma}$ by the fusion rules.

The above equation and eqs.(4.36) and (4.40) have already derived in the previous paper [5]. Here we make clear the derivation of these equations and give the explicit calculation of (4.47). If $\mathcal{O}$ includes $O_{\varepsilon}$ we have to add the following type of boundary contribution:

$$
\begin{aligned}
& \lim _{\tau \rightarrow \infty} \kappa^{2} \sum_{\Delta} \int_{-\infty}^{\infty} \frac{d p}{2 \pi} \ll \mathcal{O}_{1} \\
& \quad \times\left\{\int_{\mathrm{e}^{-\tau} \leq|z| \leq 1} d^{2} z \bar{\partial} W_{\varepsilon}(z, \bar{z}) \int_{|w| \leq|z|} d^{2} w V_{\varepsilon}(w, \bar{w})\right. \\
& \left.\quad+\int_{\mathrm{e}^{-\tau} \leq|z| \leq 1} d^{2} z V_{\varepsilon}(z, \bar{z}) \int_{|w| \leq|z|} d^{2} w \bar{\partial} W_{\varepsilon}(w, \bar{w})\right\} \\
& \quad \times \frac{-2 \pi}{H} \mathrm{e}^{-\tau H}\left|-p, \Delta \gg_{0} \ll p, \Delta\right| \mathcal{O}_{2} \gg_{0} .
\end{aligned}
$$


This contribution is calculated by using the technique developed in (4.24) and (4.42). As a result we must add the following term to r.h.s. of eq.(4.49):

$$
\begin{aligned}
-\frac{5}{2} \pi^{3} \kappa^{2}\left(\frac{\Gamma\left(\frac{1}{3}\right)}{\Gamma\left(\frac{2}{3}\right)}\right)^{3}\left[1-\frac{11}{6} \nu_{p}-\frac{209}{150} \nu+\frac{11}{12} \nu_{p}^{2}\right. \\
\left.+\frac{11}{30} \nu^{2}+\frac{11}{12} \nu_{p} \nu\right] \ll \check{\mathcal{O}}_{1} O_{I} \gg_{0} \ll O_{I} \check{\mathcal{O}}_{2} \gg_{0} .
\end{aligned}
$$

Here $\nu$ is the curvature assigned on the operator $O_{\varepsilon}$ and $\nu_{p}$ is that induced at the state $\mid-p, \Delta \gg$. The check symbol $\vee$ on $\mathcal{O}_{1}$ and $\mathcal{O}_{2}$ stands for the exclusion of the operator $O_{\varepsilon}$. The $\Gamma$-functions come from the $w$-integrals. The integrals of $p$ and $z$ are evaluated by using the saddle point method.

Let us consider the case of $\mathcal{O}=O_{\varepsilon} \Pi^{n} O_{I}$. The curvatures are assigned only on $n O_{I}$ 's $(n \geq 1)$. Then, using the result (4.53) with $\nu=0$, we get 3

$$
\begin{aligned}
& \pi \frac{3}{16 \sqrt{6}} \mu \ll O_{\varepsilon} O_{\varepsilon} \prod^{n} O_{I} \gg_{0} \\
& -\pi \frac{3}{16 \sqrt{6}}\left(n+\frac{11}{9}-\frac{55}{18} \sum_{a=1}^{n} \nu_{a}^{2}\right) \ll O_{\varepsilon} O_{\varepsilon} \prod^{n-1} O_{I} \gg_{0} \\
& -\frac{\pi}{4 \sqrt{6}} \lambda_{3} \sum_{k=0}^{n}\left[\left(\begin{array}{l}
n \\
k
\end{array}\right)-\frac{11}{12}\left(\begin{array}{l}
n-2 \\
k-1
\end{array}\right)\left(4-\sum_{a=1}^{n} \nu_{a}^{2}\right)\right] \\
& \quad \times \ll \prod^{k+1} O_{I} \gg_{0} \ll \prod^{n-k+1} O_{I} \gg_{0}=0
\end{aligned}
$$

where

$$
\lambda_{3}=\frac{1}{2} 4 \sqrt{6} \frac{5}{2} \pi^{2} \kappa^{2}\left(\frac{\Gamma\left(\frac{1}{3}\right)}{\Gamma\left(\frac{2}{3}\right)}\right)^{3}=\lambda_{1} \lambda_{2} .
$$

The factor $\frac{1}{2}$ in the middle of eq.(4.56) corrects for double counting.

${ }^{3}$ If we assign the curvature only on $O_{\varepsilon}$, we then get

$$
\begin{gathered}
\pi \frac{3}{16 \sqrt{6}} \mu \ll O_{\varepsilon} O_{\varepsilon} \prod^{n} O_{I} \gg_{0}-\pi \frac{3}{16 \sqrt{6}} n \ll O_{\varepsilon} O_{\varepsilon} \prod^{n-1} O_{I} \gg_{0} \\
+\pi \frac{2}{25 \sqrt{6}} \lambda_{3} \sum_{k=0}^{n}\left(\begin{array}{l}
n \\
k
\end{array}\right) \ll \prod \prod_{I} \gg_{0} \ll \prod^{n-k+1} O_{I} \gg_{0}=0
\end{gathered}
$$

for $n \geq 0$, where we use the formula (4.53) with $\nu=2$ and $\nu_{p}=0$. 
If we take $\mathcal{O}=O_{\varepsilon} \prod^{n} O_{\sigma}$ and assign the curvatures on $n O_{\sigma}$ 's, we get

$$
\begin{aligned}
& \pi \frac{3}{16 \sqrt{6}} \mu \ll O_{\varepsilon} O_{\varepsilon} \prod^{n} O_{\sigma} \gg_{0} \\
& -\frac{\pi}{\sqrt{6}} \lambda_{2} C_{\varepsilon \sigma \sigma} \sum_{k=0}^{n}\left[\frac{1}{2} n(n-1)-\frac{77}{30}(n-1)+\frac{11}{6}+\frac{11}{48}(n-3) \sum_{a=1}^{n} \nu_{a}^{2}\right] \\
& \quad \times \ll O_{I} O_{\varepsilon} \prod^{n-2} O_{\sigma} \gg_{0}
\end{aligned}
$$

This equation is valid for $n \geq 4$. $n=2$ is ill-defined and $n=$ odd is trivial.

Until now we introduce the three independent parameters, $\mu$ and two of $\lambda_{j}(j=1,2,3)$, which are related to the normalizations of the three scaling operators. The normalization of $O_{I}$ is fixed by the equation $-\frac{\partial}{\partial \mu} \ll$ $\mathcal{O} \gg_{0}=\ll O_{I} \mathcal{O} \gg_{0}$. If we change the normaizations of $O_{\sigma}$ and $O_{\varepsilon}$ into $\tilde{O}_{\sigma}=x O_{\sigma}$ and $\tilde{O}_{\varepsilon}=y O_{\varepsilon}$, and simultaneously $\lambda_{j}$ into $\tilde{\lambda}_{1}=\left(y / x^{2}\right) \lambda_{1}$, $\tilde{\lambda}_{2}=x^{2} y \lambda_{2}$ and $\tilde{\lambda}_{3}=y^{2} \lambda$, the recurion relations do not change the forms and they also satisfy the relation $\tilde{\lambda}_{3}=\tilde{\lambda}_{1} \tilde{\lambda}_{2}$.

\section{Solutions of Recursion Relations}

In this section we give the several solutions of the recursion relations (4.36), (4.50), (4.55) and (4.57) and compare them with the results of the matrix model. These equations are satisfied independently of the value of $\sum_{a=1}^{n} \nu_{a}^{2}$ for $n \geq 2$ because how to assign the curvature singularities is completely arbitrary. For $n=1$, however, it is fixed to the value $\sum_{a} \nu_{a}^{2}=\nu_{1}^{2}=4$.

For simplicity we here use the following notation:

$$
\ll \prod^{n_{1}} O_{I} \prod^{n_{2}} O_{\sigma} \prod^{n_{3}} O_{\varepsilon} \gg_{0}=<\prod^{n_{1}} I \prod^{n_{2}} \sigma \prod^{n_{3}} \varepsilon>.
$$


Let us write down the first several of eq.(4.36). From $n=3$ we get the following two equations:

$$
\begin{array}{r}
5 C_{\varepsilon \sigma \sigma}<\varepsilon \sigma \sigma>-3 \lambda_{1}<\sigma \sigma><\sigma \sigma I>+4 \lambda_{1}<\sigma \sigma \sigma \sigma><I>=0(5.2) \\
C_{\varepsilon \sigma \sigma}<\varepsilon \sigma \sigma>-\lambda_{1}<\sigma \sigma><\sigma \sigma I>=0(5.3)
\end{array}
$$

The second comes from the coefficients in front of $\sum_{a} \nu_{a}^{2}$. The $n=4$ equation is now trivial. The $n=5$ equation gives

$$
\begin{aligned}
& 15 C_{\varepsilon \sigma \sigma}<\varepsilon \sigma \sigma \sigma \sigma>+15 \lambda_{1}<\sigma \sigma><\sigma \sigma \sigma \sigma I> \\
& \quad-15 \lambda_{1}<\sigma \sigma \sigma \sigma><\sigma \sigma I>+4 \lambda_{1}<\sigma \sigma \sigma \sigma \sigma \sigma><I>=0
\end{aligned}
$$

and, from the coefficient in front of $\sum_{a} \nu_{a}^{2}$,

$$
-C_{\varepsilon \sigma \sigma}<\varepsilon \sigma \sigma \sigma \sigma>+\lambda_{1}<\sigma \sigma><\sigma \sigma \sigma \sigma I>+3 \lambda_{1}<\sigma \sigma \sigma \sigma><\sigma \sigma I>=0 .
$$

Next we write down the first several of eq.(4.50). From $n=2$, we obtain the following two equations:

$$
\begin{aligned}
\frac{3}{16} \mu<\varepsilon \sigma \sigma>-\frac{4}{15} \lambda_{2} C_{\varepsilon \sigma \sigma}<I>+\frac{7}{6} \lambda_{1} C_{\varepsilon \sigma \sigma}<\sigma \sigma>^{2} & =0 \\
2 \lambda_{2}<I>-5 \lambda_{1}<\sigma \sigma>^{2} & =0 .
\end{aligned}
$$

From $n=4$ we get

$$
\frac{3}{16} \mu<\varepsilon \sigma \sigma \sigma \sigma>-\frac{2}{15} \lambda_{2} C_{\varepsilon \sigma \sigma}<\sigma \sigma I>+\frac{1}{12} \lambda_{1} C_{\varepsilon \sigma \sigma}<\sigma \sigma><\sigma \sigma \sigma \sigma>=0
$$

and

$$
\lambda_{2}<\sigma \sigma I>+5 \lambda_{1}<\sigma \sigma><\sigma \sigma \sigma \sigma>=0 .
$$

Furthermore, from $n=1$ of (4.55), we obtain $\theta$

$$
-3 \mu<\varepsilon \varepsilon I>-30<\varepsilon \varepsilon>+8 \lambda_{3}<I><I I>=0 .
$$

Noting that $\langle I \mathcal{O}\rangle=-\frac{\partial}{\partial \mu}<\mathcal{O}>$ such that $<\sigma \sigma>=-(3 / 2) \mu<$ $\sigma \sigma I>, \quad<1>=-(3 / 7) \mu<I>=(9 / 28) \mu^{2}<I I>, \quad<\varepsilon \varepsilon>=-\frac{3}{5} \mu<$ $\varepsilon \varepsilon I>$ and so on, we obtain the folllowing normalization independent ratio:

$$
\frac{<\varepsilon \sigma \sigma>^{2}<1>}{<\varepsilon \varepsilon><\sigma \sigma>^{2}}=\frac{20}{7} \frac{\lambda_{1} \lambda_{2}}{\lambda_{3}} C_{\varepsilon \sigma \sigma}^{2}=\frac{5}{7} .
$$

\footnotetext{
${ }^{4}$ This equation is the same as the $n=0$ equation of (4.54) essentially.
} 
Here we use eqs.(5.6), (5.7), (5.10) and the relation (4.56) and $C_{\varepsilon \sigma \sigma}^{2}=\frac{1}{4}$. The same result is also derived by using eqs.(5.3) instead of (5.6). It is just a consistency check of the result. This value agrees with that derived by the other methods [14, 13, 6]. From (5.9) and (5.7) we get

$$
\frac{<\sigma \sigma \sigma \sigma><1>}{<\sigma \sigma\rangle^{2}}=-\frac{1}{7} .
$$

It is easily checked that the same result is given by using (5.2), (5.3) and (5.7). This also agrees with the result derived by Crnković et. al. [13]. Furthermore we get following values:

$$
\frac{<\varepsilon \sigma \sigma \sigma \sigma>^{2}<1>^{3}}{<\varepsilon \varepsilon><\sigma \sigma>^{4}}=\frac{45}{1372}
$$

by using eq.(5.5) or (5.8) and

$$
\frac{<\sigma \sigma \sigma \sigma \sigma \sigma><1>^{2}}{<\sigma \sigma>^{3}}=-\frac{15}{98}
$$

by using eq. (5.4).

From $n=4$ of eq.(4.57) we get

$$
\begin{aligned}
& \frac{3}{16} \mu<\varepsilon \varepsilon \sigma \sigma \sigma \sigma>-\frac{2}{15} \lambda_{2} C_{\varepsilon \sigma \sigma}<\varepsilon \sigma \sigma I> \\
& +\frac{1}{12} \lambda_{1} C_{\varepsilon \sigma \sigma}<\varepsilon \sigma \sigma><\sigma \sigma \sigma \sigma>+\frac{1}{12} \lambda_{1} C_{\varepsilon \sigma \sigma}<\varepsilon \sigma \sigma \sigma \sigma><\sigma \sigma> \\
& -\frac{1}{2} \lambda_{3}<\sigma \sigma \sigma \sigma I><I>+\frac{1}{3} \lambda_{3}<\sigma \sigma I>^{2}=0 .
\end{aligned}
$$

The equation coming from the coefficients in front of $\sum_{a} \nu_{a}^{2}$ does not give any new information, which is automatically satisfied by using the equations listed above. From (5.15) we obtain

$$
\frac{<\varepsilon \varepsilon \sigma \sigma \sigma \sigma><1>^{2}}{<\varepsilon \varepsilon><\sigma \sigma>^{2}}=-\frac{15}{98} .
$$

\section{Summary and Discussion}

In this paper we discussed the recursion relations of the Liouville gravity coupled to the non-bosonized Ising matter satisfying the fusion rules and 
found out a closed set of them. They have three independent parameters, $\mu$ and two of $\lambda_{j}(j=1,2,3)$ satisfying the relation $\lambda_{3}=\lambda_{1} \lambda_{2}$, which are related to the normalizations of the three scaling operators $O_{I}, O_{\sigma}$ and $O_{\varepsilon}$. We obtained some solutions and computed the normalization independent ratios. The results agree with those calculated in the other methods [13, 14, 6].

The difference from the theory using the bosonized matter is whether the gravitational descendants other than $\sigma_{1}\left(O_{\sigma}\right)=O_{\varepsilon}$ appear in the recursion relations or not. If we replace $\sigma$ and $\varepsilon$ in (2.4), (4.1-2) and (4.4-5) into the bosonized forms and introduce a screening charge in the definition of the correlation functions as in ref. [7], $\sigma_{1}\left(O_{I}\right)$ will appear and some of the OPE coefficients are changed $\mathbf{3}$. Conversely the disappearance of these operators indicates that the fusion rules are satisfied.

In exchange for the disappearance of the gravitational descendants other than $O_{\varepsilon}$, we encounter the demerit that the correlators

$$
\begin{aligned}
& \ll O_{\varepsilon} \gg_{0}, \quad \ll O_{\varepsilon} O_{I} \gg_{0}, \quad \ll O_{\varepsilon} O_{I} O_{I} \gg_{0}, \\
& \ll O_{\varepsilon} O_{\varepsilon} O_{\varepsilon} O_{\varepsilon} \gg_{0}, \quad \ll O_{\varepsilon} O_{\varepsilon} O_{\varepsilon} O_{\varepsilon} O_{I} \gg_{0}, \quad \ll O_{\varepsilon} O_{\varepsilon} O_{\sigma} O_{\sigma} \gg_{0},
\end{aligned}
$$

which have zero or positive integer $s$, and also $\ll O_{\varepsilon} O_{\varepsilon} O_{\varepsilon} \gg_{0}$, whose Liouville part diverges, become ill-defined. When the recursion relations include such a correlator they become inconsistent, or there is no solution satisfying them independently of how to assign the curvature singularities. This make impossible to calculate the correlators including more than and equal three $O_{\varepsilon}$ 's.

We do not know how to regularize these correlators. The attempt to determine the values of these correlators by imposing the requirement that the equations are naively extended to the case which include them, for instance $n=1$ of eq.(4.36), does not do well. There is no solutions satisfying such a requirement.

To make these correlators well-defined we have to go to the bosonized theory perturbed by the cosmological constant operator and one of the screening

\footnotetext{
${ }^{5}$ In the bosonized theory there exist the physical operators satisfying the $W_{\infty}$ algebra as for $R(z)[8]$. So there is no reason to use the operators of type listed in (4.4-5) in the bosonized theory.

${ }^{6}$ If we use the $W_{\infty}$ currents with the same Liouville charges as (4.4-5) instead, we furthermore encounter the gravitational descendants $\sigma_{3}\left(O_{I}\right)$ and $\sigma_{3}\left(O_{\sigma}\right)$ because the OPE's between the currents and the screening charge operator do not vanish [7].
} 
charges of matter sector [7]. Then the factor $\Gamma(-s) / n !=\Gamma(-s) / \Gamma(n+1)$ satisfying the relation $s+n+N=\chi=2$ appears in the definition of correlator, where $\Gamma(-s)$ and $1 / n !=1 / \Gamma(n+1)$ come from the zero mode integrals of the Liouville and the matter fields respectively and $N$ is the number of the scaling operators in $\ll \cdots \gg_{0}$. So the divergence of $\Gamma(-s)$ at $s \in \mathbf{Z}_{\geq 0}$ cancels out with $\Gamma(n+1)$ in the case listed in (6.1). The correlator $\ll O_{\varepsilon} O_{\varepsilon} O_{\varepsilon} \gg_{0}$ become finite in rather different way as discussed by Kitazawa in ref. [6].

\section{References}

[1] J. Distler and H. Kawai, Nucl. Phys. B321 (1989) 509; F. David, Mod Phys. Lett. A3 (1988) 1651.

[2] T. Curtright and C. Thorn, Phys. Rev. Lett. 48 (1982) 1309; N. Seiberg, Prog. Theor. Phys. Suppl. 102 (1990) 319; J. Polchinski, Proc. of the String 1990 (Texas A\& M, March 1990).

[3] M. Bershadsky and I. Klebanov, Phys. Rev. Lett. 65 (1990) 3088; Nucl. Phys. B360 (1991) 559.

[4] J. Polchinski, Nucl. Phys. B357 (1991) 241.

[5] K. Hamada, Nucl. Phys. B365 (1991) 354; Prog. Theor. Phys. Suppl. 110 (1992) 135.

[6] M. Goulian and M. Li, Phys. Rev. Lett. 66 (1991) 2051; Y. Kitazawa, Phys. Lett. B265 (1991) 262; Int. J. Mod. Phys. A7 (1992) 3403; P. DiFrancesco and D. Kutasov, Phys. Lett. B261 (1991) 385; V. Dotsenko, Mod. Phys. Lett. A6 (1991) 3601; K. Aoki and E. D’Hoker, Mod. Phys. Lett. A7 (1992) 235; N. Sakai and Y. Tanii, Prog. Theor. Phys. 86 (1991) 547; S. Govindarajan, T. Jayaraman and V. John, Phys. Rev. D48 (1993) 839; S. Yamaguchi, Mod. Phys. Lett. A8 (1993) 327.

[7] K. Hamada, Phys. Lett. B324 (1994) 141; Nucl. Phys. B413 (1994) 278.

[8] E. Witten, Nucl. Phys. B373 (1992) 187; I. Klebanov and A. Polyakov, Mod. Phys. Lett. A6 (1991) 3273; I. Klebanov, Mod. Phys. Lett. A7 
(1992) 723; N. Chair, V. Dobrev and H. Kanno, Phys. Lett. 283 (1992) 194.

[9] B. Lian and G. Zuckermann, Phys. Lett. B254 (1991) 417; Phys. Lett. B266 (1991) 21.

[10] P. Bouwknegt, J. McCarthy and K. Pilch, Comm. Math. Phys. 145 (1992) 541.

[11] K. Hamada and H. Ishikawa, "Non-linear Structures in Noncritical NSR String", Preprint YITP/U-94-16//UT-Komaba/9412//hep-th/9405130.

[12] A. Belavin, A. Polyakov and A. Zamolodchikov, Nucl. Phys. B241 (1984) 333.

[13] C̆. Crnković, P. Ginsparg and G. Moore, Phys. Lett. B237 (1990) 196.

[14] P. DiFrancesco and D. Kutasov, Nucl. Phys. B342 (1990) 589.

[15] M. Fukuma, H. Kawai and R. Nakayama, Int. J. Mod. Phys. A6 (1991) 1385; R. Dijkgraaf, E. Verlinde and H. Verlinde, Nucl. Phys. B348 (1991) 435.

[16] P. DiFrancesco, H. Saleur and J. Zuber, Nucl. Phys. B290 [FS20] (1987) 527. 